\title{
HIV's kinesin chauffeur
}
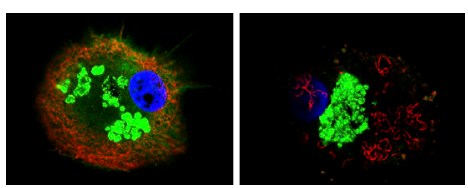

The distribution of virus-containing compartments (green) in a macrophage (left) changes after microtubules (red) are disrupted (right).

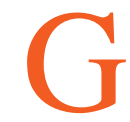
audin et al. identify a type of kinesin that ferries HIV to the plasma membrane, helping the virus escape from macrophages.

HIV reproduces inside T cells, killing them in the process. But the virus can also replicate in macrophages, which survive infection and serve as reservoirs of HIV. In T lymphocytes, new viral particles are born at the plasma membrane, but in macrophages the viruses assemble inside cytoplasmic containers called virus-containing compartments (VCCs). To break out of a macrophage, a virus particle therefore has to travel to the plasma membrane.
Gaudin et al. showed that the virus hitches a ride with the microtubule-based kinesin motor KIF3A. The researchers found that knocking down KIF3A dramatically reduced the release of HIV particles from macrophages. Yet the procedure did not have any effect on the amount of HIV escaping from $\mathrm{T}$ cells

KIF3A drives HIV along microtubules, the researchers discovered. For example, the localization of KIF3A-positive VCCs depended on microtubules. Gaudin et al. also observed KIF3A proteins and VCCs moving in tandem along the filaments. VCCs build up in cells lacking KIF3A, suggesting that their movements stall in the absence of the kinesin. How HIV exits VCCs remains uncertain, but the results indicate that inhibiting KIF3A might offer a new way to combat the virus.

Gaudin, R., et al. 2012. J. Cell Biol. http://dx.doi.org/10.1083/jcb.201201144.

\section{Only skin deep, thanks to microtubules}

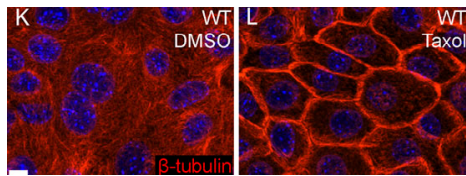

Microtubules (red) are normally spread around the cytoplasm (left), but they concentrate at the cortex after taxol treatment (right). separate chromosomes-but researchers haven't discerned all of their roles in differentiated cells. One mystery, for instance, is why microtubules accumulate at the cortex in epidermal cells. Sumigray et al. devised a system to test the functions of these microtubules in vitro. The researchers dosed layers of epidermal keratinocytes with taxol, which stabilized microtubules at the cell cortex, mimicking their organization in vivo.

Sumigray et al. found that cortical microtubules reinforce sheets of keratinocytes but not by directly providing structural support.
Instead, the fibers fortified adherens junctions that link adjacent cells, changing the composition and dynamics of these cell-cell junctions. More of the adherens junction component $\beta$-catenin gathered at the cortex, for instance. The microtubules also drew myosin II to the cortex, enabling the motor protein to apply tension and ensure that the junctions couple to the underlying actin cytoskeleton.

Adherens junctions fasten neighboring cells together, but tight junctions, another type of intercellular connection, provide a barrier that is impermeable to small molecules. The researchers found that, by modifying adherens junctions, cortical microtubules enable the tight junctions between epidermal cells to create a tighter seal. They tested this ability by injecting newborn mice with biotin, a compound that normally can't diffuse through the epidermis because of tight junctions. But biotin penetrated deeper into the skin in mice treated with nocodazole to break down their microtubules. An unanswered question, the researchers say, is how the microtubules attract myosin II to the cortex.

Sumigray, K.D., et al. 2012. J. Cell Biol. http://dx.doi.org/10.1083/jcb.201206143.

\section{$\mathrm{N}$-WASP helps cancer cells open up}

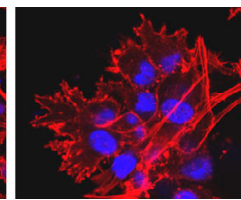

Control cancer cells stained for actin (red) produce robust extensions (left), whereas cancer cells lacking $\mathrm{N}$-WASP only manage small, spiky projections (right).

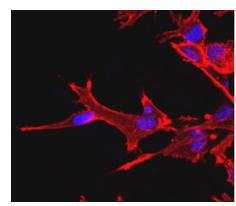

ike prisoners breaking out of jail, metastatic cancer cells tunnel through the extracellular matrix (ECM) that keeps them confined. Yu et al. reveal how an actininteracting protein helps the cells make their escape.

Tumor cells force their way through the ECM by sprouting actin-rich extensions known as invadopodia, which contain enzymes called matrix metalloproteases to dissolve the surrounding material. Previous studies implicated the actin-polymerizing protein $\mathrm{N}$-WASP in the movement of cancer cells. For example, N-WASP spurs tumor cells crawling on a two-dimensional surface to extend invadopodia.
In more realistic, three-dimensional surroundings, N-WASP also stimulated tumor cells to form invadopodia, Yu et al. showed. They found that the protein dictates a cell's temperament. Some cancer cells are leaders that burrow into the ECM. Others are followers that rely on the path cleared by the leaders. The researchers showed that cells rarely became trailblazers if they lack N-WASP.

The protein abets tumor cells in another way, helping them deploy the matrix metalloprotease MT1-MMP. The enzyme juts from the tips of invasive protrusions, and it continually cycles between the cell membrane and the lysosome. Yu et al. determined that N-WASP re-routes MT1-MMP molecules in late endosomesthe last stop before the lysosome-and sends them back to the tips of invadopodia. N-WASP even helps retain MT1-MMP at the end of an invadopodium. The actin mesh that N-WASP induces there latches onto MT1-MMP's cytoplasmic tail, holding the enzyme in place so that it can attack the ECM.

Yu, X., et al. 2012. J. Cell Biol. http://dx.doi.org/10.1083/jcb.201203025. 